\title{
Dynamic changes in myocardial architecture after reperfused acute myocardial infarction (AMI): insights from the prospective REMI study (REmodeling after Myocardial Infarction)
}

\author{
Lin Zhang ${ }^{2 *}$, Olivier Huttin ${ }^{1,2}$, Frédéric Moulin ${ }^{1}$, Pierre-Yves Marie ${ }^{1,3}$, Damien Mandry ${ }^{1,3}$ \\ From 17th Annual SCMR Scientific Sessions \\ New Orleans, LA, USA. 16-19 January 2014
}

\section{Background}

Following an acute infarction, a dynamic healing process initiates via extracellular substrate deposition and turnover, resulting in ongoing remodeling of infarct territory and global function change of left ventricle. Our prospective study was designed to investigate by CMR the dynamic changes in myocardial architecture at 2-4 days and 6 months following a reperfused AMI.

\section{Methods}

66 consecutive patients who fulfilled inclusion criteria underwent two CMRs: 2-4 days (baseline) and 6 months (follow-up) after the AMI, comprising SSFP images for analysis of left ventricular functions and late gadolinium enhancement (LGE) MRI $\left(0.1 \mathrm{mmol} / \mathrm{Kg}\right.$; DOTAREM ${ }^{\circledR}$, GUERBET, Roissy, France) for infarct analysis using a threshold method. Of 132 CMRs, 6 (4 from baseline) were non-diagnostic owing to poor EKG triggering and/or inability of the patients to hold their breath; 5 patients without evidence of infarct scar by LGE were also excluded. Hence, 57 patients ( 50 male; $56.7 \pm 13.2$ yo) were eventually analyzed. Total infarct was determined as area with signal intensity (SI) above mean $+5 \mathrm{SD}$ of remote myocardium and was further divided into peri-infarct zone (PIZ) and infarct core by introducing a 7SD threshold; microvascular obstruction was manually included into the core; a core to PIZ ratio(C/PIZ) was calculated and a transmurality score was computed using a 5 -point scale on the 16-segment model derived from the AHA model. Paired t-test was used for comparison between the two

\footnotetext{
${ }^{2}$ IADI U947, INSERM, Nancy, France

Full list of author information is available at the end of the article
MRI and Pearson's correlation coefficients were calculated to assess prediction of $\mathrm{LV}$ parameters.

\section{Results}

14 patients $(25 \%)$ had an AMI in the LAD territory; 18 patients (31.6\%) presented microvascular obstruction on LGE images at baseline. Dynamic changes of infarct indices and LV functional parameters are detailed in table 1 ; LVEF improved significantly ( $\mathrm{p}<0.0001$ ), owing to a decrease of LVESV $(\mathrm{p}<0.05)$, possibly related to stunning at subacute phase; the increase of LVEDV remained mild, possibly indicative that most of LV remodeling occurred prior to the baseline MRI. Total infarct, core and PIZ mass all diminished significantly ( $\mathrm{p}<0.0001$ for all). Both $\mathrm{C} / \mathrm{PIZ}$ and transmurality score remained identical. Both total scar and infarct core masses at baseline were predictive of LVEF, LVEDV and LVESV at follow-up (table 2), but not of the change in LV functional parameters.

\section{Conclusions}

Following reperfused AMI, our study demonstrated a significant improvement in LVEF, related to an improved contractility due to stunning in the subacute phase. Myocardial damage characteristics by LGE showed a decrease of infarct size, owing to resorption of myocardial edema, in the same amount between its two components, core and PIZ.

\section{Funding}

Grant from the French Ministry of Health Support from Laboratoire Guerbet, Roissy, France: supply of contrast medium. 
Table 1 Infarct index and LV functional parameters at baseline and follow-up.

\begin{tabular}{cccccc}
\hline & $\begin{array}{c}\text { Baseline } \\
(\mathbf{N}=\mathbf{5 7})\end{array}$ & $\begin{array}{c}\text { Follow-up } \\
(\mathbf{N}=\mathbf{5 7})\end{array}$ & Change & \%Change & P value \\
\hline LV mass, g & $97.2 \pm 19.1$ & $92.9 \pm 20.4$ & $-4.2 \pm 15.2$ & $-3.7 \pm 15.8$ & $<0.05$ \\
\hline LVEF,\% & $43.0 \pm 6.7$ & $49.4 \pm 7.7$ & $6.4 \pm 5.8$ & $15.7 \pm 15.3$ & $<0.0001$ \\
\hline LVEDV, mL & $177.4 \pm 30.4$ & $183.0 \pm 36.7$ & $5.6 \pm 22.1$ & $3.4 \pm 12.7$ & NS $(0.061)$ \\
\hline LVESV, mL & $100.3 \pm 22.7$ & $94.4 \pm 30.0$ & $-5.9 \pm 19.5$ & $-6.2 \pm 18.5$ & $<0.05$ \\
\hline Infarct mass, g & $25.4 \pm 11.8$ & $17.0 \pm 7.9$ & $-8.5 \pm 8.0$ & $-30.6 \pm 20.1$ & $<0.0001$ \\
\hline Infarct mass, \% of LV mass & $25.4 \pm 8.8$ & $17.8 \pm 6.3$ & $-7.6 \pm 6.3$ & $-27.5 \pm 20.0$ & $<0.0001$ \\
\hline Core mass, g & $12.3 \pm 6.0$ & $10.5 \pm 5.7$ & $-2.8 \pm 5.3$ & $-17.2 \pm 41.3$ & $<0.0001$ \\
\hline Core mass, \% of LV mass & $13.3 \pm 4.6$ & $10.8 \pm 4.8$ & $-2.4 \pm 4.6$ & $-14.6 \pm 37.4$ & $<0.0001$ \\
\hline PIZ mass, g & $9.6 \pm 4.5$ & $6.5 \pm 2.9$ & $-3.2 \pm 3.5$ & $-26.6 \pm 26.3$ & $<0.0001$ \\
\hline PIZ mass, \% of LV mass & $9.7 \pm 3.6$ & $6.9 \pm 2.7$ & $-2.7 \pm 3.2$ & $-22.8 \pm 28.2$ & $<0.0001$ \\
\hline Core/PIZ & $1.6 \pm 0.8$ & $1.7 \pm 0.8$ & $0.1 \pm 1.0$ & $28.4 \pm 80.5$ & NS(0.405) \\
\hline Transmurality score & $1.30 \pm 0.31$ & $1.21 \pm 0.43$ & $-0.1 \pm 0.45$ & $-3.53 \pm 35.68$ & NS(0.118) \\
\hline
\end{tabular}

LV indicates left ventricular; LVEF, LV ejection fraction; LVEDV, LV end-diastolic volume; LVESV, LV end-systolic volume; PIZ, peri-infarct zone; NS, no significant

Table 2 Pearson's correlation coefficients between infarct indices and LV remodeling.

\begin{tabular}{|c|c|c|c|c|c|c|}
\hline & $\operatorname{LVEF}_{2}, \%$ & $\Delta$ LVEF, $\%$ & LVEDV $_{2}, \mathrm{~mL}$ & $\Delta$ LVEDV, $\mathrm{mL}$ & LVESV $_{2}, \mathrm{~mL}$ & $\Delta$ LVESV, $\mathrm{mL}$ \\
\hline Infarct mass $1, \mathrm{~g}$ & $-0.426^{* *}$ & -0.096 & $0.598^{* *}$ & 0.228 & $0.601^{* *}$ & 0.207 \\
\hline Infarct mass $2, \mathrm{~g}$ & $-0.486^{* *}$ & -0.198 & $0.615^{* *}$ & $0.420^{* *}$ & $0.625^{* *}$ & $0.358^{* *}$ \\
\hline Core mass $_{1}, \mathrm{~g}$ & $-0.408^{* *}$ & -0.157 & $0.500^{* *}$ & 0.137 & $0.518^{* *}$ & 0.189 \\
\hline Core mass $_{2}, \mathrm{~g}$ & $-0.497^{* *}$ & -0.248 & $0.561^{* *}$ & $0.491^{* *}$ & $0.598^{* *}$ & $0.440^{* *}$ \\
\hline PIZ mass, 19 & -0.144 & 0.08 & $0.519^{* *}$ & 0.111 & $0.436^{* *}$ & 0.100 \\
\hline $\mathrm{PIZ}$ mass $_{2}, \mathrm{~g}$ & $-0.341^{* *}$ & -0.05 & $0.561^{* *}$ & 0.175 & $0.518^{* *}$ & 0.108 \\
\hline Core/PIZ & -0.23 & -0.167 & -0.109 & -0.008 & 0.012 & 0.053 \\
\hline Core/PIZ 2 & $-0.291^{*}$ & -0.191 & 0.129 & $0.415^{* *}$ & 0.209 & $0.359^{* *}$ \\
\hline \%change Core/PIZ & -0.198 & -0.04 & $0.281^{*}$ & $0.409^{* *}$ & $0.299^{*}$ & $0.319^{*}$ \\
\hline Transmurality score $_{1}$ & -0.274 & 0.011 & $0.362^{* *}$ & 0.123 & $0.331^{*}$ & 0.007 \\
\hline Transmurality score $_{2}$ & -0.160 & 0.089 & 0.238 & 0.205 & 0.204 & 0.000 \\
\hline
\end{tabular}

${ }^{*} p<0.05 ;{ }^{* *} p<0.01$; subscript 1 and 2 indicate baseline and follow-up data, respectively.

\section{Authors' details}

${ }^{1}$ CHU Nancy, Nancy, France. ${ }^{2}$ IADI U947, INSERM, Nancy, France. ${ }^{3}$ Universite de Lorraine, Nancy, France.

Published: 16 January 2014

\section{doi:10.1186/1532-429X-16-S1-P223}

Cite this article as: Zhang et al:: Dynamic changes in myocardial architecture after reperfused acute myocardial infarction (AMI): insights from the prospective REMI study (REmodeling after Myocardial Infarction). Journal of Cardiovascular Magnetic Resonance 2014 16(Suppl 1): P223.
Submit your next manuscript to BioMed Central and take full advantage of:

- Convenient online submission

- Thorough peer review

- No space constraints or color figure charges

- Immediate publication on acceptance

- Inclusion in PubMed, CAS, Scopus and Google Scholar

- Research which is freely available for redistribution

Submit your manuscript at www.biomedcentral.com/submit 